\title{
Video Article \\ Implementation of a Permeable Membrane Insert-based Infection System to Study the Effects of Secreted Bacterial Toxins on Mammalian Host Cells
}

\author{
Rebecca A. Flaherty ${ }^{1}$, Shaun W. Lee ${ }^{1}$ \\ ${ }^{1}$ Department of Biological Sciences, Eck Institute for Global Health, University of Notre Dame
}

Correspondence to: Shaun W. Lee at Shaun.W.Lee.310@nd.edu

URL: https://www.jove.com/video/54406

DOI: doi:10.3791/54406

Keywords: Immunology, Issue 114, Bacterial toxins, permeable membrane insert-based infection system, Streptolysin S, host-pathogen response, Group A Streptococcus, signal transduction, host cytotoxicity

Date Published: 8/19/2016

Citation: Flaherty, R.A., Lee, S.W. Implementation of a Permeable Membrane Insert-based Infection System to Study the Effects of Secreted Bacterial Toxins on Mammalian Host Cells. J. Vis. Exp. (114), e54406, doi:10.3791/54406 (2016).

\section{Abstract}

Many bacterial pathogens secrete potent toxins to aid in the destruction of host tissue, to initiate signaling changes in host cells or to manipulate immune system responses during the course of infection. Though methods have been developed to successfully purify and produce many of these important virulence factors, there are still many bacterial toxins whose unique structure or extensive post-translational modifications make them difficult to purify and study in in vitro systems. Furthermore, even when pure toxin can be obtained, there are many challenges associated with studying the specific effects of a toxin under relevant physiological conditions. Most in vitro cell culture models designed to assess the effects of secreted bacterial toxins on host cells involve incubating host cells with a one-time dose of toxin. Such methods poorly approximate what host cells actually experience during an infection, where toxin is continually produced by bacterial cells and allowed to accumulate gradually during the course of infection. This protocol describes the design of a permeable membrane insert-based bacterial infection system to study the effects of Streptolysin S, a potent toxin produced by Group A Streptococcus, on human epithelial keratinocytes. This system more closely mimics the natural physiological environment during an infection than methods where pure toxin or bacterial supernatants are directly applied to host cells. Importantly, this method also eliminates the bias of host responses that are due to direct contact between the bacteria and host cells. This system has been utilized to effectively assess the effects of Streptolysin S (SLS) on host membrane integrity, cellular viability, and cellular signaling responses. This technique can be readily applied to the study of other secreted virulence factors on a variety of mammalian host cell types to investigate the specific role of a secreted bacterial factor during the course of infection.

\section{Video Link}

The video component of this article can be found at https://www.jove.com/video/54406/

\section{Introduction}

Understanding the function of bacterial virulence factors in the context of host cell infection is a major focus of bacterial pathogenesis research. Many bacterial pathogens actively secrete toxins and other soluble factors to aid in the destruction of host tissue, to initiate signaling changes in host cells or to manipulate immune system responses during the course of infection ${ }^{1-10}$. Though methods have been developed to successfully purify and produce many of these important virulence factors for study, some bacterial products have unique structures or extensive posttranslational modifications that make them recalcitrant candidates for purification methods and thus cannot be studied in isolation using in vitro systems. For example, Group A Streptococcus, the bacterial pathogen responsible for a myriad of infections ranging from pharyngitis to necrotizing fasciitis and toxic shock syndrome, produces a secreted bacterial toxin known as Streptolysin S (SLS) ${ }^{11-17}$. This ribosomally produced peptide is encoded by the Streptolysin S associated gene (sag) cluster, and the mature product is estimated to be $2.7 \mathrm{kDa}$ in size ${ }^{14-17}$. The protoxin, encoded by sagA, is modified post-translationally by several enzymes (SagB, SagC, and SagD) to produce the mature, functional form $^{15-17}$. The complexity of these post-translational modifications coupled with the toxin's unusual amino acid sequence have rendered the toxin incompatible with all purification and structure elucidation efforts that have been attempted to date ${ }^{15,17}$. These challenges have complicated efforts to determine the specific role of this toxin in host pathogenesis.

In cases where the preparation of purified toxins or other secreted factors is either complex or not possible, mechanisms to elucidate the function of these products have traditionally been studied through the preparation of filtered bacterial supernatants, which are then applied to host cells $^{18-20}$. There are several challenges associated with this technique. First, many of these secreted factors, including SLS, do not maintain maximal or consistent activity when stored and applied to host cells at a later time. Additionally, when supernatants are collected at a single time point and then applied to host cells, it is difficult to determine physiological relevance and to draw direct conclusions about the natural infection process, where secreted factors are allowed to accumulate during the course of infection to a physiologically relevant concentration. This second challenge applies not only to the use of bacterial supernatants, but also to the use of purified toxins in host cell studies ${ }^{1-3,8}$. To address these issues, a permeable membrane insert-based infection system has been developed to assess the effects of SLS on host cells in a manner that maintains optimal toxin activity and also eliminates the variable of direct contact between bacteria and host cells. In this system, human epithelial cells are grown in a monolayer in the bottom chamber of a two chamber well, and bacteria are introduced into the upper chamber of the same well. A porous membrane $(0.4 \mu \mathrm{m}$ pores $)$ separates the upper and lower chambers, allowing secreted factors to be exchanged between the 
two chambers but preventing the passage of bacteria. This system has allowed for effective evaluation of host responses that are solely due to sustained secreted bacterial components while eliminating any responses that may occur through direct contact during Group A Streptococcal infection. Though other secreted bacterial factors besides SLS can also pass through the porous membrane, the use of an isogenic mutant panel including wild-type (WT), an SLS-knockout $(\triangle \operatorname{sag} A)$ and a sagA complemented strain $(\Delta \operatorname{sag} A+\operatorname{sag} A)$ allows for precise evaluation of host responses that are strictly SLS-dependent ${ }^{21}$.

Although similar permeable membrane insert systems have been employed for the study of secreted factors involved in viral infections, cancer biology, and immune cell migration ${ }^{22-26}$, few studies involving bacterial interactions with host cells have utilized this approach ${ }^{6,27,28}$. Even studies that have employed such systems to explore interactions between bacteria and host cells have primarily focused on migration of inflammatory cells or bacteria through an epithelial or endothelial monolayer plated on the permeable membrane insert. The permeable membrane insertbased infection system described herein is a simple and effective method that relies on the separation of bacteria and host cells via a porous membrane to assess the effects of the secreted toxin, SLS, on host membrane integrity, cellular viability, cellular signal transduction, and secreted host cell factors. This technique can be adapted to the study of other secreted virulence factors on a variety of mammalian host cell types to investigate the role of a specific bacterial factor over the course of an infection.

Protocol

\section{Bacterial Culture}

1. Generate isogenic mutant strains to be used for the study of the specific secreted factor of interest ${ }^{21}$.

Note: The GASM1T1 5448 strains utilized for these experiments included WT GAS', a sagA $\triangle$ cat SLS-deficient mutant (abbreviated in text as $\triangle \operatorname{sag} A$ ), and a $\operatorname{sag} A$ complemented strain (abbreviated in text as $\triangle \operatorname{sag} A+\operatorname{sag} A)^{21}$.

2. Prepare overnight liquid cultures of bacterial strains of interest. Grow GAS M1T1 5448 strains in $-10 \mathrm{ml}$ of Todd-Hewitt broth at $37{ }^{\circ} \mathrm{C}$ for 16-20 hr prior to infecting human cells.

3. Centrifuge overnight bacterial cultures $(2,400 \mathrm{rcf}$ for $10 \mathrm{~min})$ and resuspend in fresh bacterial medium. Note: Resuspension in the same volume as the overnight cultures were grown in (5-10 ml) generally produces a desirable initial optical density.

4. Normalize bacterial cultures to equal starting concentrations by diluting the re-suspended cultures in additional bacterial medium until the same optical density at $600 \mathrm{~nm}\left(\mathrm{OD}_{600}\right)$ is obtained for all strains to be tested $\left(\mathrm{OD}_{600}\right.$ of approximately 1.0 is recommended for convenience). Note: In these studies, stationary phase bacterial cultures were used to infect host cells immediately following normalization. However, as some strains of bacteria exit stationary phase nonsynchronously it may be more appropriate to begin host infections with log phase cultures if this is found to be the case for the strains of interest.

5. Prior to further analysis, perform a colony counting assay to determine the colony forming units (CFU) per milliliter present in the starting cultures so that the multiplicity of infection (MOI), or ratio of bacteria per host cell, can be determined.

1. Prepare $1 \mathrm{ml}$ serial dilutions of bacterial cultures that have been normalized to the desired optical density in phosphate buffered saline (PBS) or appropriate bacterial growth medium (Todd-Hewitt broth was used in these studies). Prepare dilutions ranging from $10^{-1}$ to $10^{-6}$ to produce at least one set of agar plates with countable colonies (30-300 colonies). Perform all dilutions in triplicate.

2. Transfer $100 \mu \mathrm{l}$ of each serial dilution onto an agar plate containing appropriate medium for the bacterial species being analyzed. Note: Todd-Hewitt agar was used in these studies.

3. Use a glass or plastic bacterial spreader to evenly distribute the $100 \mu \mathrm{l}$ aliquot across the entire surface of the agar plate, and continue spreading until the liquid has been absorbed into the agar. Perform under flame using sterile technique.

4. Incubate the agar plates at $37^{\circ} \mathrm{C}$ overnight or until countable colonies appear.

5. Count the colonies that grow on each plate and calculate the CFU/ml in the original culture by the following formula: number of colonies $x$ inverse of the serial dilution / culture volume plated in milliliters.

e.g. $\left(200\right.$ colonies $\times 1 / 10^{-5}$ dilution $) / 0.1 \mathrm{ml}$ plated $=2.0 \times 10^{8} \mathrm{CFU} / \mathrm{ml}$

\section{Host Cell Culture}

1. Obtain an appropriate host cell line for the desired analyses.

Note: HaCaT human epithelial keratinocytes ${ }^{29}$, a kind gift from V. Nizet, were used for these studies.

1. Maintain HaCaT cells in $100 \mathrm{~mm}$ culture dishes in Dulbecco's Modified Eagle's Medium (DMEM) with $10 \%$ heat inactivated fetal bovine serum (FBS). Incubate at $37^{\circ} \mathrm{C}$ with $5 \% \mathrm{CO}_{2}$.

\section{Host Cell Infection with Permeable Membrane Insert System}

1. Plate cells in appropriate tissue culture dishes and allow them to grow until they reach $90 \%$ confluency.

Note: The HaCaT cells used in these studies typically reach confluency within 2-3 days after plating.

1. For lysate collection (e.g. signaling analysis and adenosine triphosphate (ATP) determination assays), plate HaCaT cells in 6 well dishes at a seeding density of approximately $3 \times 10^{5}$ cells/well.

2. For immunofluorescence imaging experiments, plate cells on sterile glass coverslips within 6 well dishes at a seeding density of approximately $3 \times 10^{5}$ cells/well.

3. For ethidium homodimer membrane permeabilization and lactate dehydrogenase (LDH) release assays, plate HaCaT cells in 24 well dishes at a seeding density of approximately $5 \times 10^{4}$ cells/well.

2. Immediately prior to treatment, wash cells with $1 \mathrm{x}$ sterile PBS. 
3. Apply fresh growth medium to cells. For the conditions described here, apply $2 \mathrm{ml}$ medium per well for 6 well plates or $0.5 \mathrm{ml}$ medium per well for 24 well plates. If pharmacological treatments are being tested, mix with fresh medium and apply during this step.

Note: Some assays may require alternative media. For example, as phenol red and high serum concentrations interfere with the LDH release assay, phenol red-free DMEM supplemented with $1 \%$ bovine serum albumin (BSA) (w/v), $2 \mathrm{mM}$ L-glutamine, and $1 \mathrm{mM}$ sodium pyruvate was utilized for these experiments.

4. After fresh medium has been applied, carefully place a sterile $0.4 \mu \mathrm{m}$ permeable membrane insert in each well. Perform this step in a laminar flow hood, and use sterile forceps to transfer the permeable membrane insert into each well.

5. Apply fresh cell culture medium to the upper chamber of each well according to the manufacturer's instructions. For the conditions described here, apply $1 \mathrm{ml}$ medium per well for 6 well plates or $0.1 \mathrm{ml}$ medium per well for 24 well plates.

6. Apply an appropriate volume of normalized bacterial cultures (see section 1) to the upper chamber of the permeable membrane insert system. Use bacterial medium for uninfected control treatments. For the results described here, add $25 \mu \mathrm{l}$ of the normalized cultures per chamber for 24 well plates and add $100 \mu \mathrm{l}$ of the cultures per chamber for 6 well plates.

Note: In these studies, wild-type or mutant GAS was applied to host cells at a MOI of 10. MOI was calculated based on the final eukaryotic cell numbers (e.g. $2 \times 10^{6}$ cells/well), such that 10 times as many bacteria were added per host cell at the beginning of the infection period (e.g. $2 \times 10^{7} \mathrm{CFU}$ per well). Appropriate MOI will vary with different bacterial species and with the desired follow-up analyses.

Note: In addition to using bacterial medium for uninfected controls, other useful controls for certain applications of this technique may include heat-killed bacteria or spent bacterial medium for comparison.

7. Incubate infected cells at $37^{\circ} \mathrm{C}$ with $5 \% \mathrm{CO}_{2}$.

\section{Sample Collection}

1. To collect cell culture medium, host cell lysates, or glass coverslips with intact cells for follow-up analyses, begin by carefully removing the permeable membrane insert with sterile forceps.

2. For Assessment of Secreted Host Protiens:

1. Collect the medium from the lower chamber into $1.5 \mathrm{ml}$ tubes. Avoid disturbing the monolayer during collection.

2. Centrifuge samples at $14,000 \mathrm{rcf}$ for $10 \mathrm{~min}$ at $4{ }^{\circ} \mathrm{C}$ to remove cellular debris.

3. Remove all but $50 \mu \mathrm{l}$ of the supernatant to a fresh $1.5 \mathrm{ml}$ tube and store at $-20^{\circ} \mathrm{C}$ or use immediately.

3. For Assessment of Host Cell Lystates:

1. Gently aspirate the medium above the monolayer of host cells. Avoid disturbing the monolayer, as this may result in sample loss.

2. Rinse cells once with $1 x$ PBS.

3. Gently aspirate PBS. Immediately apply an appropriate volume of ice-cold lysis buffer to achieve the desired protein concentration, and incubate the samples on ice for $15 \mathrm{~min}$. Apply between $200 \mu \mathrm{l}$ and $350 \mu \mathrm{l}$ of lysis buffer per well of each 6 well plate to achieve protein concentrations between 0.5 and $1.5 \mathrm{mg} / \mathrm{ml}$.

Note: The lysis buffer used in these studies was composed of deionized water, $1 \%(\mathrm{v} / \mathrm{v})$ Nonidet P40 Substitute, a phosphatase inhibitor cocktail, and a protease inhibitor cocktail. Specific reagents are listed in the Materials and Equipment section.

4. Use a cell scraper to detach the cells from the plate surface of each well and pipette the entire contents of each well into a $1.5 \mathrm{ml}$ tube.

5. Centrifuge samples at $14,000 \mathrm{rcf}$ for $20 \mathrm{~min}$ at $4{ }^{\circ} \mathrm{C}$.

6. To assess soluble lysate components, remove the supernatant to a fresh tube and store at $-20{ }^{\circ} \mathrm{C}$ or use immediately.

7. To assess nuclear or other insoluble lysate components, reserve the pellet and store at $-20{ }^{\circ} \mathrm{C}$ or use immediately.

4. For Assessment by Immunofluorescence Imaging:

1. Aspirate medium and wash cells in $1 x$ cold PBS.

2. Fix cells overnight in $4 \%$ paraformaldehyde (PFA) solution in PBS (w/v). Note: In these studies, $1 \mathrm{ml}$ of PFA was added per well of each 6 well plate.

\section{Suggested Applications}

1. Host Signal Transduction Analysis by SDS-Page and Western Blotting

1. Collect host cell lysates as described in section 4.3 .

2. Determine the protein concentration of each sample lysate by bicinchoninic acid (BCA) assay or equivalent using protein standards ${ }^{30}$

3. Normalize protein concentrations between samples prior to loading and running the samples on a $4-15 \%$ polyacrylamide gel or appropriate alternative ${ }^{31}$.

1. Normalize sample concentrations by pipetting the same protein amount from each sample (e.g. $20 \mu \mathrm{g})$ into a new $1.5 \mathrm{ml}$ tube and adding variable volumes of lysis buffer (buffer volume = total volume - sample volume added) to each tube to make the total volume (e.g. $50 \mu \mathrm{l})$ and final protein concentration equivalent across samples.

4. Transfer samples to a polyvinylidene fluoride (PVDF) membrane (or equivalent). For the results described here, transfer samples at 25 volts for $2 \mathrm{hr}$ before increasing the voltage to 70 volts for an additional $45 \mathrm{~min}$. Use transfer buffer composed of $200 \mathrm{mM}$ Tris base, 1.5 $\mathrm{M}$ glycine and $20 \%$ methanol $(\mathrm{v} / \mathrm{v})$ in deionized water.

5. Block membranes in $5 \%$ BSA $+0.1 \%$ Tween 20 in Tris buffered saline (TBS). Adjust accordingly based on manufacturer recommendations for the antibody to be used.

6. Incubate membranes with primary antibodies overnight at $4{ }^{\circ} \mathrm{C}$. Use at manufacturer recommended dilution.

7. Wash membranes for $1.5 \mathrm{hr}$ in TBS $+0.1 \%$ Tween 20 ; refresh buffer every $10-15 \mathrm{~min}$.

8. Incubate with Horseradish Peroxidase (HRP)-conjugated secondary antibody at a dilution of 1:5,000 for $1.5 \mathrm{hr}$ at room temperature.

9. Wash membranes for $1.5 \mathrm{hr}$ in TBS $+0.1 \%$ Tween 20; refresh buffer every $10-15 \mathrm{~min}$. 
10. Incubate membranes with chemiluminescence detection reagent prior to developing on film according to the manufacturer's instructions. Other detection methods may be used as desired.

2. Host Cell Immunofluorescence Staining and Imaging

1. Fix coverslips containing host cells as described in section 4.4 .

2. Remove PFA solution and wash coverslips containing treated cells two times in PBS, aspirating between washes. Note: PFA is highly toxic and must be disposed of appropriately.

3. Block the coverslips for $2 \mathrm{hr}$ at room temperature in PBS with $1 \%(\mathrm{w} / \mathrm{v})$ normal goat serum, $2 \%(\mathrm{v} / \mathrm{v})$ Triton X-100, and $0.5 \%(\mathrm{v} / \mathrm{v})$ Tween 20.

4. Wash coverslips with PBS for $30 \mathrm{~min}$, refreshing the buffer every $10 \mathrm{~min}$.

5. Incubate the coverslips with primary antibody at a 1:50 ratio (or as recommended by manufacturer) in blocking solution overnight at 4 ${ }^{\circ} \mathrm{C}$.

6. Wash the coverslips for $1.5 \mathrm{hr}$ in PBS, refreshing the buffer every $30 \mathrm{~min}$.

7. Incubate the coverslips for $2 \mathrm{hr}$ at room temperature in secondary antibody (Goat anti-rabbit IgG AlexaFluor488 was used for these studies) using a 1:200 ratio of antibody to blocking solution.

8. Wash coverslips for $1 \mathrm{hr}$, refreshing the buffer every $20 \mathrm{~min}$.

9. Apply desired stains.

Note: These studies utilized DAPI (nuclear stain) and rhodamine-phalloidin (actin stain) at ratios of 1:1,000 in blocking solution.

10. Incubate coverslips with nuclear and actin stains for $30 \mathrm{~min}$ at room temperature.

11. Wash coverslips in PBS for 30 min at room temperature, refreshing the buffer every $10 \mathrm{~min}$

12. Mount the coverslips on glass slides using mounting medium.

13. Allow coverslips to set on the slides overnight prior to sealing and imaging. For the results described here, collect images on a fluorescence microscope using a standard 20X objective. Use ImageJ and microscope imaging software to process the captured images.

3. Ethidium Homodimer Cell Death Assay

1. Aspirate medium from each well and wash cells twice with sterile PBS.

2. Apply $4 \mu \mathrm{M}$ ethidium homodimer-1 in PBS.

3. Incubate cells at room temperature for $30 \mathrm{~min}$ in the dark.

4. Determine the level of fluorescence using a plate reader set to $528 \mathrm{~nm}$ excitation and $617 \mathrm{~nm}$ emission with a cutoff value of $590 \mathrm{~nm}$.

5. Add $0.1 \%(\mathrm{w} / \mathrm{v})$ Saponin to each well following the initial reading and allow the plate to incubate for an additional $20 \mathrm{~min}$ at room temperature (with rocking) before reading the plate a second time at the same settings.

6. Determine percent membrane permeabilization individually for each well by dividing the initial fluorescence reading (post-treatment) by the second fluorescence reading (post-Saponin).

4. ATP Determination Assay

1. Collect lysates as described in section 4.3 above.

2. Normalize protein levels in the lysates via BCA assay or similar ${ }^{30}$.

3. Determine cellular ATP levels using a luminescence-based ATP determination kit or equivalent according to the manufacturer's instructions.

4. Normalize treated values to the corresponding uninfected control samples.

5. LDH Release Assay

1. Following infection, collect supernatants as described in section 4.3.

2. Evaluate $\mathrm{LDH}$ release using a cytotoxicity detection kit for $\mathrm{LDH}$ release according to the manufacturer's instructions. 


\section{Representative Results}

The permeable membrane insert-based infection system protocol developed for the study of secreted bacterial factors is detailed in Figure 1. This system relies on the separation of bacteria and host cells via a porous membrane to assess the effects of secreted bacterial factors, in this case Streptolysin S (SLS), on host responses such as host membrane integrity, cellular viability, cellular signal transduction, and secreted host cell factors. Figure 2 provides representative Western Blot data demonstrating that this system may be used to assess changes in the activation of contact-independent host signaling proteins. Specifically, the representative data show significantly enhanced p38 MAPK activation in the presence of SLS-producing GAS strains. This system can also be applied to visualize the effects of secreted bacterial factors on host protein localization by immunofluorescence microscopy (Figure 3). The data show SLS-dependent activation of the key inflammatory mediator Nuclear Factor kappa B (NFkB), which translocates from the cytoplasm to the nucleus upon activation ${ }^{21}$. The results in Figures $\mathbf{2}$ and $\mathbf{3}$ indicate that both SLS-dependent inflammatory signaling responses do not require direct contact between the bacteria and host cells. As previous experiments have already demonstrated SLS-dependent p38 and NFKB activation in direct infection models ${ }^{21}$, similar levels of p38 or NFKB activation among the three GAS strains in the permeable membrane insert-based system would have indicated that the response required direct contact between the bacteria and host cells. Figure $\mathbf{4}$ demonstrates that this infection system may be applied to assess toxin-dependent changes in host cytotoxicity via ethidium homodimer and LDH release assays. This is evidenced by the significant increase in both membrane permeabilization and in the release of LDH from host cells exposed to GAS strains containing SLS compared to uninfected cells or cells exposed to the SLS-deficient strain. These changes in cytotoxicity are not immediate, as significant effects are not evident until $12 \mathrm{hr}$ post infection in the case of membrane permeabilization and $16 \mathrm{hr}$ in the case of LDH release. The representative data illustrate the importance of selecting appropriate time points and infection conditions for the evaluation of these host responses. In addition to allowing for the assessment of host signaling changes and cytotoxicity, the permeable membrane insert-based infection system is applicable to the study of metabolic changes such as accurate determination of host ATP levels by preventing bacterial contamination of host cell lysates (Figure 5). These data show a significant loss of keratinocyte ATP in response to GAS infection by 16 hours post infection, with enhanced ATP loss in the presence of SLS. These results are consistent with the observed toxin-dependent increases in host stress-response signaling and cytotoxicity.

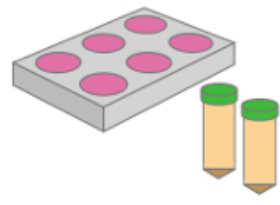

Prior to experiment:

1. Seed host cells at appropriate density.

2. Begin overnight cultures of bacteria.

Day of experiment:

1. Wash cells and refresh media.

2. Insert Transwells and add media to upper chamber.

3. Determine CFU of bacterial cultures.
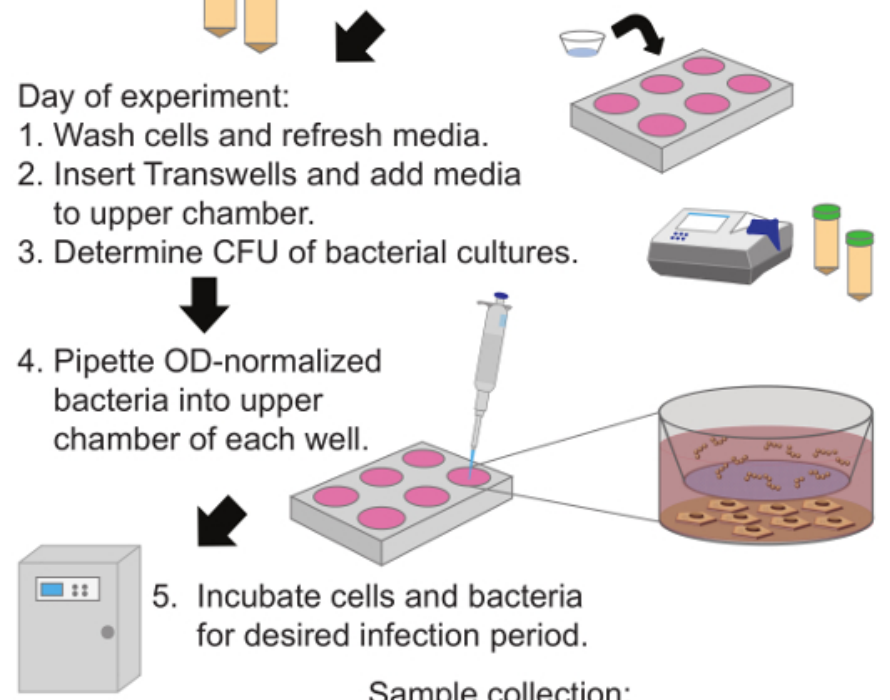

5. Incubate cells and bacteria for desired infection period.

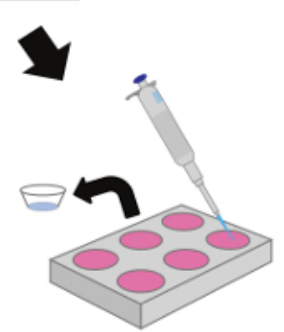

Sample collection:

1. Remove Transwell inserts.

2. Collect cell culture supernatants.

3. Wash cells with PBS.

4. Prepare host cells for desired application:
a. Lysate analysis
b. Immunofluorescence imaging
c. Staining for cytotoxicity

Figure 1: Diagram of Permeable Membrane Insert-Based Infection System Protocol to Assess the Effects of Secreted Bacterial Factors on Host Cells. Human keratinocytes are plated in the bottom compartment and grown to $90 \%$ confluence. A collagen coated membrane with 0.4 $\mu \mathrm{m}$ pores separates the upper and lower chambers, and bacteria are added to the upper chamber of the permeable membrane insert system for the desired infection period. Cell culture supernatants and host cells may be collected following the infection period and utilized for a variety of analyses. Please click here to view a larger version of this figure. 
A

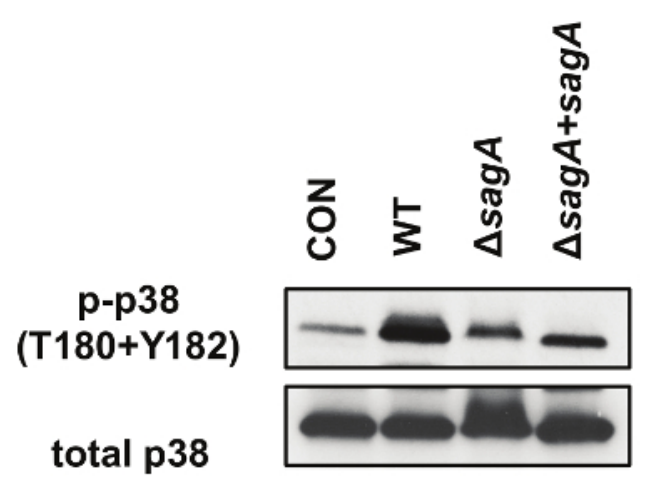

B

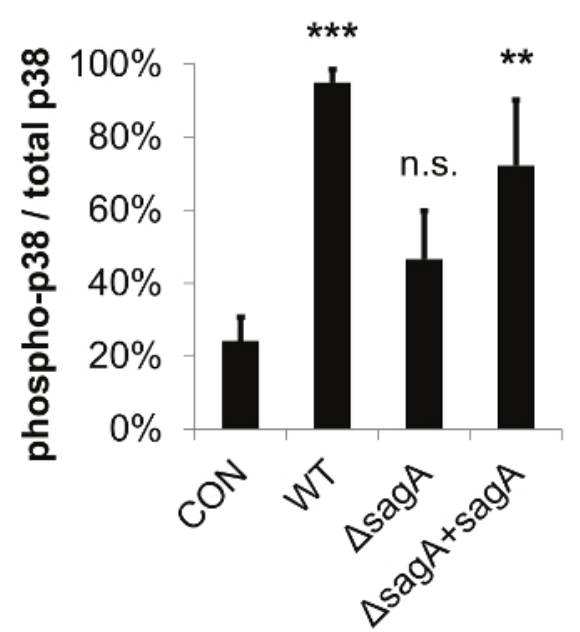

Figure 2: The Permeable Membrane Insert-Based Infection System can be Utilized for Host Cell Lysate Analysis by SDS-PAGE and Western Blotting. The representative data demonstrate that SLS enhances activation of the p38 MAPK pathway in infected keratinocytes. (A) HaCaTs were infected with GAS for $7 \mathrm{hr}$ via the permeable membrane insert infection system (at MOI $=10$ ) and lysates were assessed for activation of p38. (B) Densitometry from three independent Western Blots was performed to quantify the relative activation of p38 in response to GAS'infection. Averages from three biological replicates are shown, with error bars representing standard deviation. Relative activation of p38 is represented as phosphorylated/total protein level. Statistical significance was determined compared to uninfected cells. The overall $p$-value was determined by ANOVA $(p=0.0063)$. Dunnett's tests were performed post hoc to compare each condition to the corresponding uninfected control mean. ${ }^{*}, p=0.01-0.05 ;{ }^{* *}, p=0.001-0.01 ;{ }^{* * *}, p=0.0001-0.001 ;{ }^{* * *}, p<0.0001$. This figure has been modified from Flaherty et al. $2015^{21}$.

Please click here to view a larger version of this figure. 


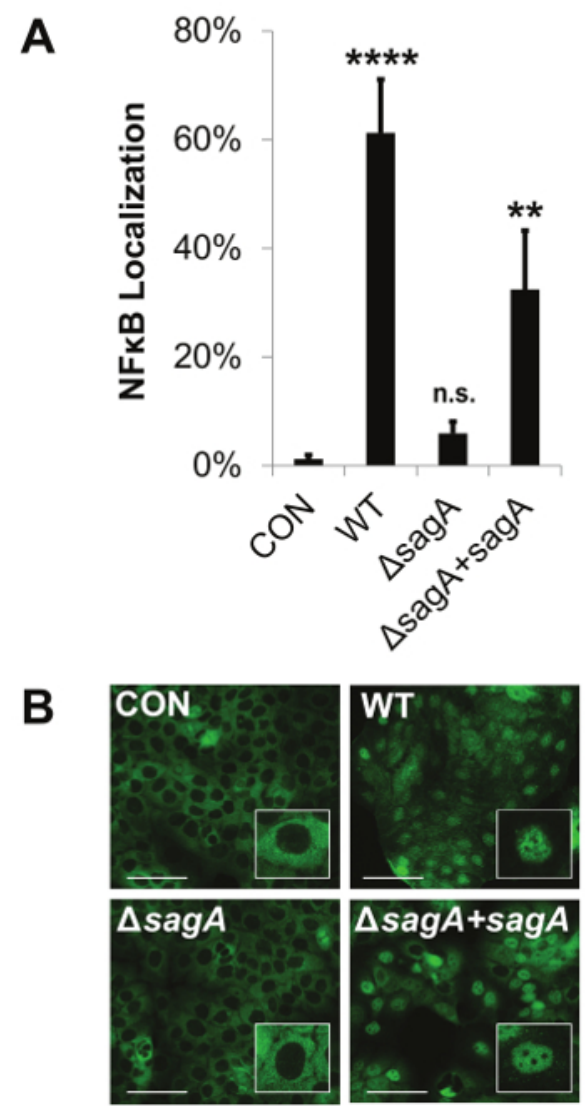

Figure 3: The Permeable Membrane Insert-Based Infection System Allows for the Visualization of Host Signaling Changes by Immunofluorescence Microscopy. The representative data show that Streptolysin S enhances pro-inflammatory signaling through activation of NFKB. HaCaT human keratinocytes were infected with GAS at an MOI of 10 for $8 \mathrm{hr}$ using the permeable membrane insert-based infection system. (A and B) Nuclear localization of NFKB was assessed by immunofluorescence imaging. The percentage of nuclear localized cells was calculated by counting the number of cells in which NFKB had translocated from the cytoplasm to the nucleus for a given field and dividing that number by the total number of cells for the same field. Scale bars indicate $100 \mu \mathrm{m}$. (A) The average of three biological replicates are represented for each condition with error bars representing standard deviation. The overall $p$-value was determined by ANOVA; $p<0.0001$. Dunnett's tests were performed to compare each condition to the corresponding uninfected control condition. ${ }^{*}, p=0.01-0.05 ;{ }^{* *}, p=0.001-0.01 ;{ }^{* * *}, p=$ $0.0001-0.001 ;{ }^{* * * *}, p<0.0001$. This figure has been modified from Flaherty et al. $2015^{21}$. Please click here to view a larger version of this figure. 


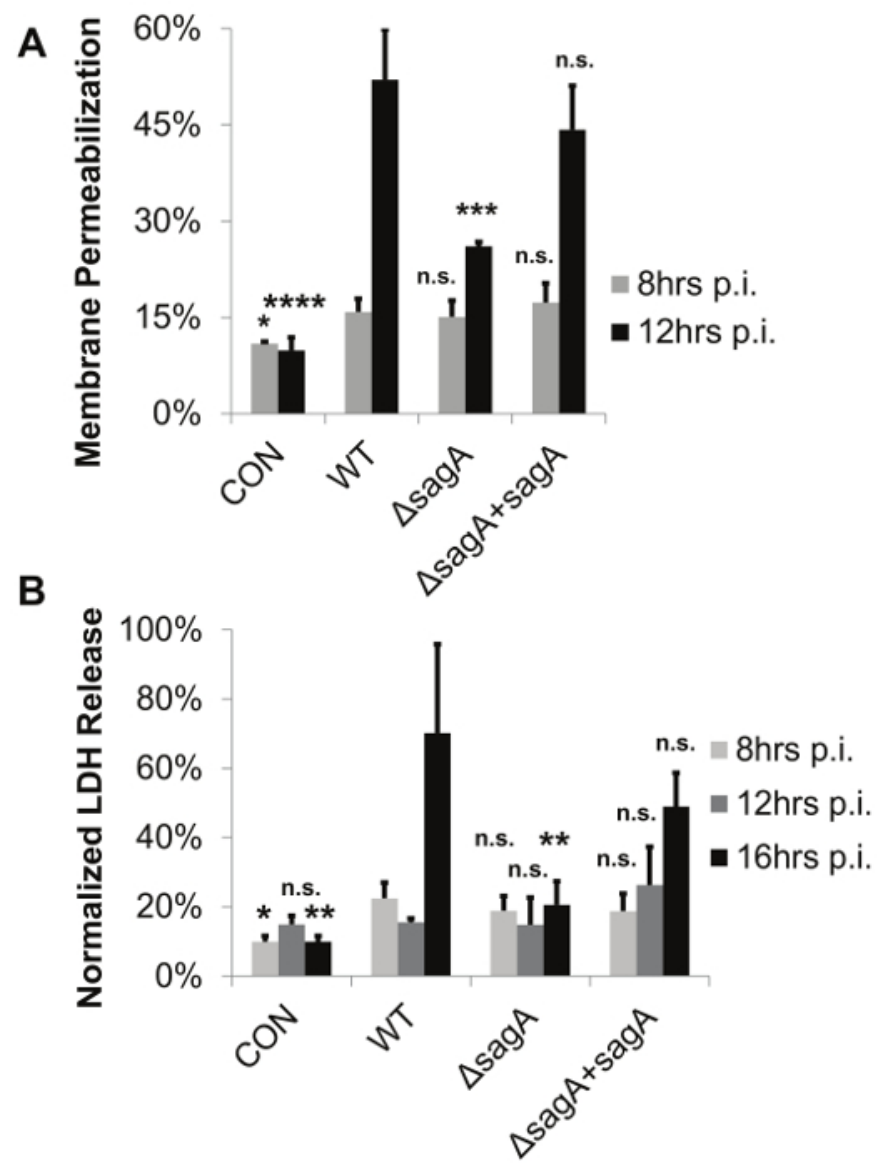

Figure 4: The Permeable Membrane Insert-Based Infection System Allows for the Determination of Bacterial-Mediated Membrane Permeabilization and Cytotoxicity in the Absence of Direct Contact between Bacteria and Host Cells. The representative data demonstrate that keratinocyte viability decreases in the presence of active SLS toxin. GAS-induced cell death was assessed in HaCaT cells in the presence of WT, SLS-deficient or sagA complemented GAS. Keratinocytes were exposed to GAS using the permeable membrane insertbased infection system for 8-16 hr at an MOI of 10. (A) Viability was assessed by ethidium homodimer assay or (B) LDH release assay. In both panels, 3 replicates are averaged and error bars represent standard deviation. Significance for each time point was determined by ANOVA (A) $8 \mathrm{hr}, \mathrm{p}=0.0241 ; 12 \mathrm{hr}, \mathrm{p}<0.0001$ (B) $8 \mathrm{hr}, \mathrm{p}=0.0287 ; 12 \mathrm{hr}, \mathrm{p}=0.1977 ; 16 \mathrm{hr}, \mathrm{p}=0.0031$. Dunnett's tests were performed to compare means from each condition to the wild-type infection for the corresponding time point. ${ }^{*}, p=0.01-0.05 ;{ }^{* *}, p=0.001-0.01 ;{ }^{* * *}, p=0.0001-0.001 ;{ }^{* * *}, p$ $<0.0001$. This figure has been modified from Flaherty et al. $2015^{2 \uparrow}$. Please click here to view a larger version of this figure. 


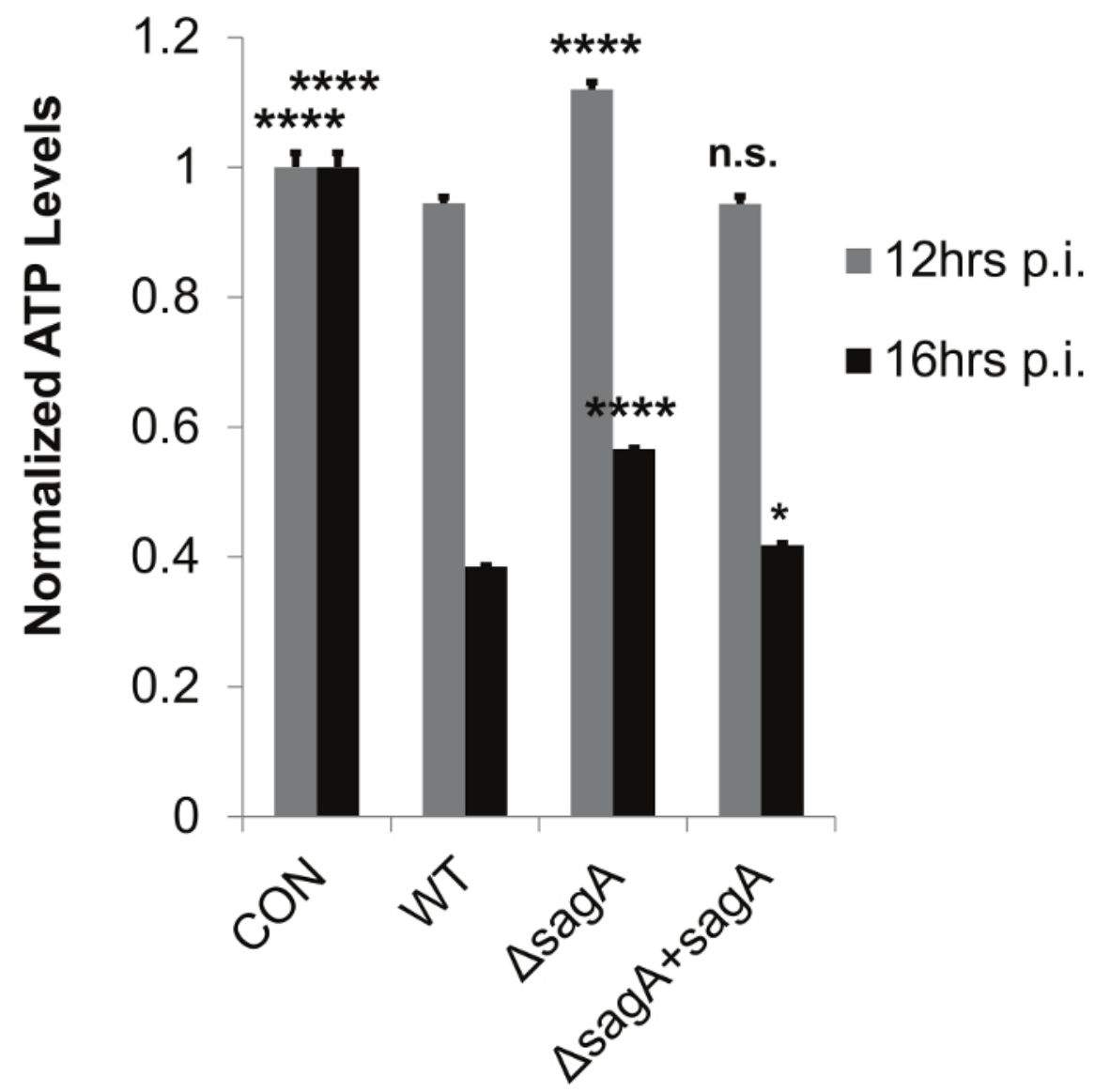

Figure 5: The Permeable Membrane Insert-Based Infection System Allows for Accurate Determination of Host ATP Levels by Preventing Bacterial Contamination of Host Cell Lysates. The representative data demonstrate SLS-dependent loss of ATP during Group A Streptococcal infection. HaCaT cells were infected with GAS for 8-16 hr using the permeable membrane insert-based infection system. Technical replicates $(n=3)$ from one representative biological replicate $\left(2 \times 10^{6}\right.$ cells per sample) were averaged for each condition, with error bars representing standard deviation. The overall $p$-values were determined by ANOVA $(12 \mathrm{hr}, \mathrm{p}<0.0001 ; 16 \mathrm{hr}, \mathrm{p}<0.0001)$. Dunnett's tests were performed to compare each condition with wild-type infection for the corresponding time point. ${ }^{*}, p=0.01-0.05 ;{ }^{*}, p=0.001-0.01 ;{ }^{* *}, p=$ $0.0001-0.001 ;{ }^{* \star \star}, p<0.0001$. This figure has been modified from Flaherty et al. $2015^{21}$. Please click here to view a larger version of this figure.

\section{Discussion}

Herein is described a method using a permeable membrane insert-based infection system to examine the effects of the bacterial toxin Streptolysin S on human epithelial keratinocytes in an in vitro system. This protocol can be adapted for the study of other secreted bacterial virulence factors as well as alternative host cell types. This recently developed system provides several advantages over experimental methods that utilize purified toxins or filtered bacterial supernatants ${ }^{1-3,8,18-20}$. The permeable membrane insert-based system provides a constantly maintained dose of the relevant bacterial toxin to host cells as it is produced, which allows maximal toxin activity to be maintained and also increases consistency between experiments. Additionally, this system more closely mimics physiological conditions by allowing the secreted factor to accumulate over time as the infection progresses and by eliminating the need to arbitrarily select specific toxin concentrations to apply to host cells. Furthermore, this system would also provide means for investigators to assess whether a bacterial factor of interest is delivered to host cells in a contact-dependent manner. Contact-dependence is often tested through the generation of isogenic bacterial mutants deficient in adherence or through the use of reagents that prevent adherence. The system described here would provide a simple alternative to complement or replace these traditional approaches.

In addition to the tested applications described in section 5 of the protocol, other applications to which this infection system could be easily adapted include the collection of samples for cytokine arrays and ELISA assays. In both of these cases, a similar protocol to that described for $\mathrm{LDH}$ release assays may be followed. Though not shown here, this system has been used to effectively collect host cell samples to be analyzed by flow cytometry. In this application, the permeable membrane insert is removed following the infection period, the cells are washed to remove cell culture medium, and trypsin is applied to allow collection of the cells for analysis. The physical separation of bacteria from host cells is particularly useful for this application because it helps to prevent the formation of large aggregates comprised of adhered bacteria and host cells that could otherwise interfere with accurate cell counting by the flow cytometer.

Though very consistent host responses have been observed when comparing the results of the permeable membrane insert-based infection studies with traditional direct infection studies, some notable differences in the kinetics of these host responses have been observed ${ }^{21}$. For example, changes in host signaling and membrane-based cytotoxicity take 30-50\% longer to occur in the permeable membrane insert-based infection model than corresponding direct infection models. Because the permeable membrane insert-based system requires medium to be 
applied to the upper and lower compartments of each well, the system developed for the studies described here required a $30 \%$ increase in total medium volume per well compared to corresponding direct infection models used previously ${ }^{21}$. This difference in total medium volume, coupled with the increased distance between bacteria and host cells when direct contact is prohibited, likely increases the time it takes for SLS to diffuse through the medium to reach host cells and elicit the observed effects. Additionally, the permeable membrane insert-based system removes many GAS virulence factors that are likely to contribute to host cell damage; the absence of these additional factors in this system is also likely to contribute to the delay in host cell death and initiation of host signaling events compared to direct infection ${ }^{21}$. These factors should be considered when designing experiments to assess other secreted bacterial components.

To identify the most meaningful conditions for testing the effects of secreted bacterial factors on host cells, testing a range of time points for each application of the permeable membrane insert-based infection system is recommended. It is important to consider under what conditions the specific virulence factor of interest is optimally produced (e.g. log phase, stationary phase, in response to certain environmental signals, etc.) in order to successfully observe its effects. In experiments focused on evaluating changes in host signaling proteins, it was necessary to select time points that allowed adequate time for SLS to reach host cells and to be produced in sufficient amounts to induce signaling changes ${ }^{21}$. At the same time, assessment of changes in host signaling needed to be conducted prior to SLS-induced membrane permeabilization, as this effect complicates the collection of host cell lysates for analysis. Cell death induced by SLS could be optimally observed in both direct and permeable membrane insert-based infection models several hours after the initiation of the reported signaling events ${ }^{21}$. Furthermore, in selecting time points of interest, it is also important to ensure that the bacteria being studied are unable to penetrate the porous insert membrane during the period of study. The production of certain bacterial components over an extended period may disrupt the integrity of the membrane and allow the passage of bacteria to the lower compartment. To determine whether this is a potential problem in the system of interest, the bacterial strains to be studied can be applied to the upper chamber of the permeable membrane insert-based system at a range of time points. At each time point, the insert can be carefully removed and the medium from the bottom chamber can be collected and utilized in a standard colony counting assay (see section 1.5). If no colonies are formed from the cell culture medium in the lower compartment, the insert membrane may be assumed to be effectively preventing the passage of bacteria at the time point and bacterial load tested.

Another experimental design component that is likely to require optimization for the effective analysis of secreted bacterial factors is the multiplicity of infection (MOI). The $\mathrm{MOI}$ refers to the ratio of bacterial cells per host cell, and is therefore influenced by host cell confluency at the time of infection and by the number of bacterial colony forming units (CFU) applied to the cells. In these studies, keratinocyte cells were grown to $90 \%$ confluency, which allowed these cells to form a cohesive monolayer with intact tight junctions. Thoughtful consideration of the physiological organization of the host cells to be studied is necessary to select an appropriate confluency for infection experiments. Once an appropriate number of host cells per well is determined, a suitable bacterial CFU can be calculated based off the desired MOI. In the studies described here, GAS was applied to host cells at a MOI of 10. Appropriate MOI will vary with different bacteria and with the desired follow-up analyses. A low beginning $\mathrm{MOI}$ is typically more physiologically relevant and allows for the bacterial factor of interest to accumulate slowly enough to capture subtle changes in host cell signaling before dramatic changes in host cell viability are evident. Higher MOls are used in many studies assessing cytotoxicity, but this effect can also be achieved by beginning with a low $\mathrm{MOI}$ and allowing the infection to progress for a longer period of time. It is important to determine an accurate CFU to optical density corollary for all bacterial strains to be tested, as isogenic mutants may have an altered growth rate compared to wild-type bacteria and may therefore require that a higher or lower CFU be added per well to ensure an appropriate comparison of the host response between strains. In cases where the mammalian host cells being studied are capable of killing bacteria or inhibiting their growth or if nonsynchronous growth between bacterial strains is suspected, it may also be useful to perform studies to assess the final bacterial load at the end of the infection period. This could be accomplished by collecting the contents of the permeable insert and performing a colony counting assay similar to that described in section 1.5 of the procedure.

Selecting appropriately sized wells for the desired assay is also important for obtaining optimal results. Permeable membrane inserts are available in a variety of sizes, but the most consistent results for the studies shown here were observed using inserts designed for 24 well and 6 well tissue culture plates. These insert sizes are relatively easy to handle with sterile forceps, and ease of manipulation is critical in preventing unwanted transfer of bacteria from the upper compartment to the lower compartment of the well. For experiments involving the collection of host cell lysates, 6 well dishes provide an appropriate cell number per condition for most analyses and are large enough to accommodate cell scrapers for sample collection. For cytotoxicity assays in which the host cells remain adherent and are labeled with a fluorescent or colorometric dye that can be measured on a plate reader (e.g. ethidium homodimer assay, trypan blue exclusion assay), use of a 24 well plate with the corresponding inserts is recommended. For experiments in which cell culture medium will be collected and analyzed (e.g. LDH release, cytokine studies) either the 24 well or 6 well plates may be used, though the smaller wells typically provide adequate sample volumes for these analyses and minimize use of the required reagents. Overall, the method is highly versatile and may be adapted as needed to prepare samples for numerous follow-up applications.

\section{Disclosures}

The authors have nothing to disclose.

\section{Acknowledgements}

We thank our fellow Lee Lab colleagues, who provided valuable insight and expertise that greatly assisted this research. This work was supported by the Young Innovator Award from the National Institute of Health, awarded to S.W. Lee (NIH-1DP2OD008468-01). R.A. Flaherty is supported by the Albertus Magnus Fellowship provided by Dr. Robert C. Boguslaski, and a teaching assistantship through the Eck Institute for Global Health at the University of Notre Dame. 


\section{References}

1. Wiles, T. J., Dhakal, B. K., Eto, D. S., \& Mulvey, M. A. Inactivation of host Akt/protein kinase B signaling by bacterial pore-forming toxins. Mol Biol Cell. 19 (4), 1427-1438 (2008).

2. Kao, C. Y., Los, F. C. O., et al. Global functional analyses of cellular responses to pore-forming toxins. PLoS Pathog. 7 (3), e1001314 (2011).

3. Ma, X., Chang, W., Zhang, C., Zhou, X., \& Yu, F. Staphylococcal Panton-Valentine Leukocidin Induces Pro-Inflammatory Cytokine Production and Nuclear Factor-Kappa B Activation in Neutrophils. PLoS ONE. 7 (4), e34970 (2012).

4. Sumitomo, T., Nakata, M., et al. Streptolysin S contributes to group A streptococcal translocation across an epithelial barrier. J Biol Chem. 286 (4), 2750-2761 (2011).

5. Miyoshi-Akiyama, T., Takamatsu, D., Koyanagi, M., Zhao, J., Imanishi, K., \& Uchiyama, T. Cytocidal effect of Streptococcus pyogenes on mouse neutrophils in vivo and the critical role of streptolysin S. J Infect Dis. 192 (1), 107-116 (2005).

6. Goldmann, O., Sastalla, I., Wos-oxley, M., Rohde, M., \& Medina, E. Streptococcus pyogenes induces oncosis in macrophages through the activation of an inflammatory programmed cell death pathway. Cell Microbiol. 11 (1), 138-155 (2009).

7. Lin, A., Loughman, J. A., Zinselmeyer, B. H., Miller, M. J., \& Caparon, M. G. Streptolysin S inhibits neutrophil recruitment during the early stages of Streptococcus pyogenes infection. Infect Immun. 77 (11), 5190-5201 (2009).

8. Ratner, A. J., Hippe, K. R., Aguilar, J. L., Bender, M. H., Nelson, A. L., \& Weiser, J. N. Epithelial cells are sensitive detectors of bacterial poreforming toxins. J Biol Chem. 281 (18), 12994-12998 (2006).

9. Stassen, M., Müller, C., et al. The streptococcal exotoxin streptolysin O activates mast cells to produce tumor necrosis factor alpha by p38 mitogen-activated protein kinase- and protein kinase C-dependent pathways. Infect Immun. 71 (11), 6171-6177 (2003).

10. Porta, H., Cancino-Rodezno, A., Soberón, M., \& Bravo, A. Role of MAPK p38 in the cellular responses to pore-forming toxins. Peptides. 32 (3), 601-606 (2011).

11. Walker, M. J., Barnett, T. C., et al. Disease manifestations and pathogenic mechanisms of group a Streptococcus. Clin Microbiol Rev. 27 (2), 264-301 (2014).

12. Carapetis, J. R., Steer, A. C., Mulholland, E. K., \& Weber, M. The global burden of group A streptococcal diseases. Lancet Infect Dis. 5 (11), 685-694 (2005).

13. Cunningham, M. W. Pathogenesis of group A streptococcal infections. Clin Microbiol Rev. 13 (3), 470-511 (2000).

14. Molloy, E. M., Cotter, P. D., Hill, C., Mitchell, D. A., \& Ross, R. P. Streptolysin S-like virulence factors: the continuing sagA. Nature Rev Microbiol. 9 (9), 670-681 (2011).

15. Datta, V., Myskowski, S. M., et al. Mutational analysis of the group A streptococcal operon encoding streptolysin S and its virulence role in invasive infection. Mol Microbiol. 56 (3), 681-695 (2005).

16. Lee, S. W., Mitchell, D. A., et al. Discovery of a widely distributed toxin biosynthetic gene cluster. Proc Natl Acad Sci USA. 105 (15), 5879-5884 (2008).

17. Mitchell, D. A., Lee, S. W., et al. Structural and functional dissection of the heterocyclic peptide cytotoxin streptolysin S. J Biol Chem. 284 (19), 13004-13012 (2009).

18. Bárcena-Uribarri, I., Benz, R., et al. Characterization of Clostridium perfringens TpeL Toxin Gene Carriage, Production, Cytotoxic Contributions, and Trypsin Sensitivity. Biochim Biophys Acta Biomem. 1848 (6), 2369-2381 (2015).

19. Bárcena-Uribarri, I., Benz, R., Winterhalter, M., Zakharian, E., \& Balashova, N. Pore forming activity of the potent RTX-toxin produced by pediatric pathogen Kingella kingae: Characterization and comparison to other RTX-family members. Biochim Biophys Acta Biomem. 1848 (7), 1536-1544 (2015).

20. Carr, A., Sledjeski, D. D., Podbielski, A., Boyle, M. D., \& Kreikemeyer, B. Similarities between complement-mediated and streptolysin Smediated hemolysis. J Biol Chem. 276 (45), 41790-4176 (2001).

21. Flaherty, R. A., Puricelli, J. M., Higashi, D. L., Park, C. J., \& Lee, S. W. Streptolysin S Promotes Programmed Cell Death and Enhances Inflammatory Signaling in Epithelial Keratinocytes during Group A Streptococcus Infection. Infect Immun. 83 (10), 4118-4133 (2015).

22. Guedon, J. T., Luo, K., Zhang, H., \& Markham, R. B. Monoclonal and Single Domain Antibodies Targeting $\beta$-Integrin Subunits Block Sexual Transmission of HIV-1 in In Vitro and In Vivo Model Systems. J Acquir Immune Defic Syndr. 69 (3), 278-285 (2015).

23. Hu, D., Zhou, J., Wang, F., Shi, H., Li, Y., \& Li, B. HPV-16 E6/E7 promotes cell migration and invasion in cervical cancer via regulating cadherin switch in vitro and in vivo. Arch Gynecol Obstet. 292 (6), 1345-1354 (2015).

24. Gangl, K., Waltl, E. E., et al. Infection with Rhinovirus Facilitates Allergen Penetration Across a Respiratory Epithelial Cell Layer. Int Arch Allergy Immunol. 166 (4), 291-296 (2015).

25. Peng, X., Zhang, Q., Zeng, Y., Li, J., Wang, L., \& Ai, P. Evodiamine inhibits the migration and invasion of nasopharyngeal carcinoma cells in vitro via repressing MMP-2 expression. Cancer Chemother Pharmacol. 76 (6), 1173-1184 (2015).

26. Zheng, Y., Guo, J., et al. FoxM1 transactivates PTTG1 and promotes colorectal cancer cell migration and invasion. BMC Med Genomics. 8, $49(2015)$.

27. Losa, D., Köhler, T., et al. Airway Epithelial Cell Integrity Protects from Cytotoxicity of Pseudomonas aeruginosa Quorum-Sensing Signals. Am J Respir Cell Mol Biol. 53 (2), 265-275 (2015).

28. Cook, K. W., Letley, D. P., et al. CCL20/CCR6-mediated migration of regulatory T cells to the Helicobacter pylori-infected human gastric mucosa. Gut. 63 (10), 1550-1559 (2014).

29. Boukamp, P., Petrussevska, R. T., Breitkreutz, D., Hornung, J., Markham, A., \& Fusenig, N. E. Normal keratinization in a spontaneously immortalized aneuploid human keratinocyte cell line. J Cell Biol. 106 (3), 761-771 (1988).

30. Krieg, R. C., Dong, Y., Schwamborn, K., \& Knuechel, R. Protein quantification and its tolerance for different interfering reagents using the BCA-method with regard to 2D SDS PAGE. J Biochem Biophys Methods. 65 (1), 13-19 (2005).

31. Brunelle, J. L., \& Green, R. One-dimensional SDS-polyacrylamide gel electrophoresis (1D SDS-PAGE). Methods Enzymol. 541, 151-159 (2014). 